\title{
A Broad Survey on Performance Analysis of Number Plate Recognition from Stationary Images and Video Sequences
}

\author{
Tahir Khan $^{1 *}$, Dr J S Yadav ${ }^{2}$, Dr Dheeraj Agarwal ${ }^{3}$ \\ ${ }^{1}$ Maulana Azad National Institute of Technology, BHOPAL (MP) INDIA \\ ${ }^{2}$ Maulana Azad National Institute of Technology, BHOPAL (MP) INDIA \\ ${ }^{3}$ Maulana Azad National Institute of Technology, BHOPAL (MP) INDIA \\ "Corresponding author E-mail: prof.tahirkhan@gmail.com
}

\begin{abstract}
Licensed Number plate recognition plays vital role in smart cities for maintaining Law \& Order and traffic management. NPR based system mainly involves four stages namely 1) Image capture \& Pre-Processing 2) Number plate area determination 3) Character Segmentations 4) Recognition of all character. This survey paper extensively analyzed the method of extraction of number plate, its platform, performance and execution time. With the development of Multilayer Perceptron Network accuracy and time in image processing has been achieved up to a great instant. Hence this analysis will help the precise assessment in establishing research and enable developers to assess which strategies are aggressive in present environment.
\end{abstract}

Keywords: Image Processing: Multilayer Perceptron: Neural Network: Performance Analysis: Optical Character Recognition (OCR)

\section{Introduction}

Information technology is rapidly advancing with respect to automated system. Transport surveillance has been developed tremendously from simple to intelligent transport system which industry needed in the era of development. There are many needs due to which NPR system has evolved and now equipped with the latest technology. Requirement like traffic surveillance, Toll Payments, parking fees payments, stolen car recovery \& on road Fatalities etc are some very urgent needs from society which require an intelligent solution to resolve number plate recognition rapidly. Number plate image segmentation is most challenging task in image processing for image analysis pattern recognition and computer vision [1][2].

\section{Number Plate Recognition}

Number Plate Recognition (NPR) based system mainly involves four stages namely 1) Image capture \& Pre-Processing 2) Number plate area determination 3) Character Segmentations 4) Recognition of all character. Image processing technique is mainly used in first three stages on stationary image and frames sequence from video, whose calculation depend upon on the error recognition and true recognition rate and lastly recognition is done through various approaches [5].

\subsection{Image Capture and Pre-Processing}

After Image Capture, pre-processing of number plate is performed through many ways [5]. It is required to improve quality of image such as complex backgrounds, low lightening, damage or dirty plates (defects on the plate surface) shadow and to remove noise from image.

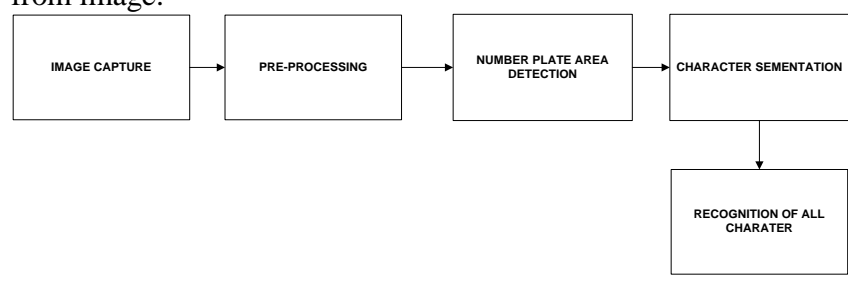

Fig. 1: Basic Block Diagram of NPR System

The Grey level methods are recommended widely because the use of color-based method has become very limited [6]. Otsu binarization method is used mainly for two reasons, one to highlight characters and other to suppress background noise [3]. Different techniques have been developed utilizing vector quantization, the Hough transform, Gabor transform and wavelet examination for this reason [7]. There are numerous areas with high densities of dark pixels on a white background in the NP region and recognized it utilizing adaptive vector quantization, Hough Transform on image edges [8].

\subsection{Number Plate Area Determination}

Our objective at this phase is to identify the Number Plate area from the original image utilizing edge-based image processing scheme. To handle this various method are developed for image enhancement like morphological methods, Canny edge detection, OCR etc [14]. Optical character recognition OCR is used for number plate area determination. It recognizes printed or written character by computer. OCR engines turn images of machine printed characters into machine-readable characters. This is done by photo scanning of text character by character, analysis of the scanned in image and then conversion of character image into 
character code. As there are many ways for NP area determination like Vertical edge detection for finding pixels with sharp changes in brightness along with skew correction for tilted image.
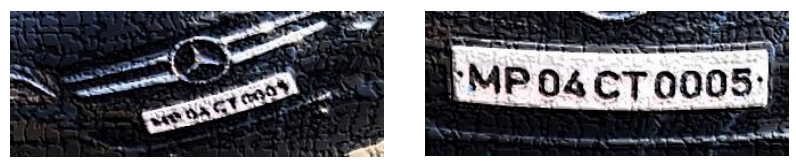

(a)

(b)

Fig. 2: (a) Tilted Image (b) Skew corrected image after Horizontal and Vertical correction

\subsection{Character Segmentation}

Character segmentation is one of the most important step in NPR for all further steps. If it fails, NPR immediately gives the wrong results. In Threshold method image of number plate is converted into black and white ("zero" \& "One") using threshold value. If pixel value is lower than threshold value, the new pixel value will be ' 0 ' else ' 1 ' for pixel with the value above the threshold value Otsu's Method [3]. Character segmentation is required to perform character recognition, which fully depends upon isolated character. Characters which are wrongly segmented are not likely to be identified successfully. Most of the recognition errors in NPR are not because of missing identification of character but due to segmentation error. In [17] they have utilized top-hat transformation and otsu threshold for segmentation.

\subsection{Recognition of All Character}

The last stage of NPR should be able to manage successfully any difficulty that may occur due to incorrectly segmented character from character segmentation. For character recognition many techniques like template matching using correlation, common pattern-matching technique, numerous algorithms, artificial neural network and computational intelligence architecture are used.

\section{Template Matching}

Template matching is a high level machine vision technique for detection and recognition of objects in computer vision community. Many techniques like Grayscale based matching, Naïve template matching, image correlation matching pattern correlation matching, edge based matching, image correlation matching etc have developed [20].
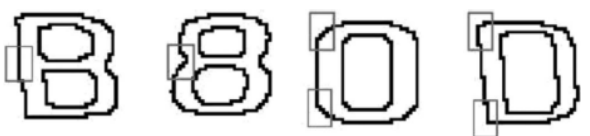

Fig 3: Distinguishing parts of ambiguous characters

\section{Neural Network}

Artificial intelligence (AI), deep learning, and neural networks represent exciting and powerful machine learning-based techniques used to resolve many challenges from the real-world [11]. A Neural Network (NN), is a numerical model or computational model that tries to repeat the structure or potentially practical neural networks. NN is an information organizing perspective that is blended by the way trademark material frameworks, for instance, the brain, process information. The key piece of this viewpoint is the novel structure of the data preparing system. It is made out of a broad number of fundamentally interconnected preparing portions (neurons) working as one to manage particular issues like pattern matching [9]. NNs, similar to individuals, learn by case. A NN is proposed for a particular application, for example, handwriting recognition, number plate recognition, diagram recognition or information game-plan, through a learning technique [4].

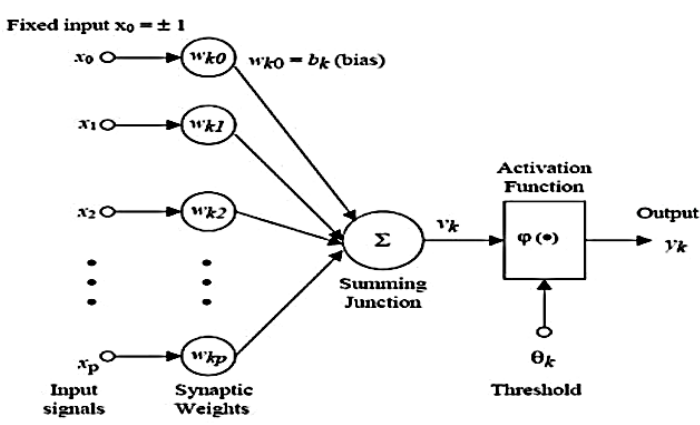

Fig 4: Artificial Neural Network

\section{Back Propagation Network}

BPN is used for training artificial neural network with mathematical foundation [18]. Back-propagation algorithm is intended for multilayer perception (MLP) to settle and register its parameters to limit a suitable cost capacity of its yield. The approach utilized in multi-layer forward network with gradient descent based on deltalearning rule alongside a guided training scheme [4]. The readiness algorithm of back propagation incorporates four stages:

1. Initialization of weights (some little arbitrary esteem)

2. $\quad$ Feed forward

3. Back propagation of mistakes (target - output)

4. Update the weights and inclinations

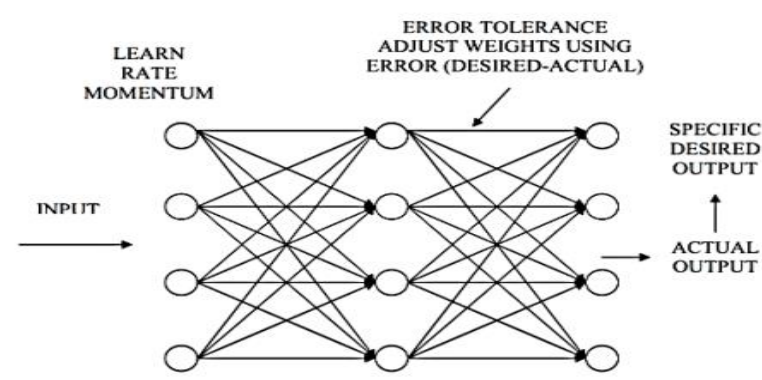

Fig 5: (a) Back Propagation network

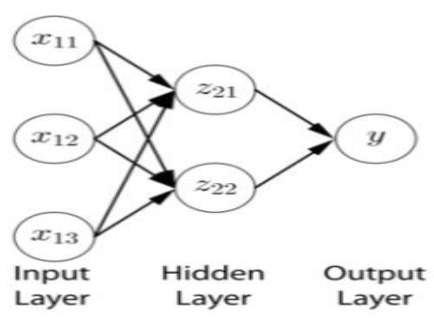

Fig 5: (b) Hidden Layer

The back propagation architecture is multilayered (hidden layer in between input layer and output layer) and designed in manage forward outline with the ability to send back the bungle between the genuine yield and the target yield regards. Each layer contains interconnected components that carry on like delicate straight classifiers. Every component figures a weighted entirety of the sources of info and changes the aggregate through a nonlinear squashing capacity. The learning is satisfied by cycles of altering the weights on every connection in order to limit a target work that was prevalently the mean square mistake between Back propagation was utilized to ascertain the slope of the target work. 


\section{Related Work}

Y. Shima [9] reported a technique that is fit for extraction of number plate region in the car image fetched at various distances from its back side. The system examines the input image and distinguishes the region of the number plate. The number plate location is fetched by utilizing dilation morphology and the number of connected components in the region and scoring based on the height width ratio. In this image extractor pre-trained convolution neural network is used as a classifier, support vector machine is used. The algorithm was implemented by $\mathrm{C}++$ in morphological image processing in MATLAB and deep learning with pre-trained Convolution neural network. Edge detection for Morphological image processing was depending upon on connected components. Based on the outcomes, approaches are suited for rear image. The number plate extraction, rate of success was $89.7 \%$ among 126 sample images.

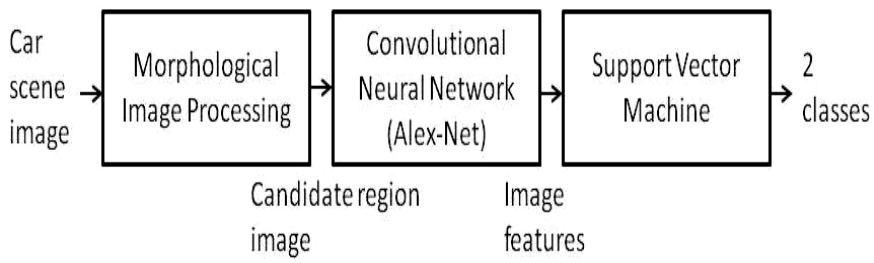

Fig. 6 : A flow of number plate image extraction using deep learning.

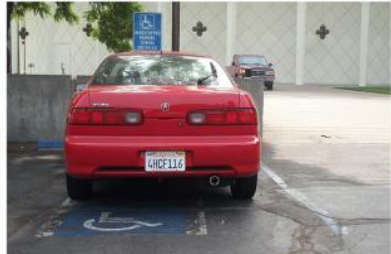

(a) Input color image

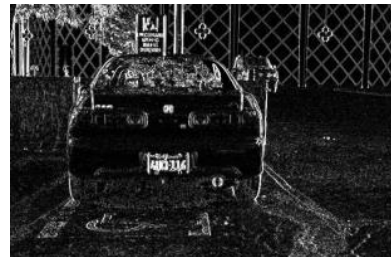

(c) Detected edge Image

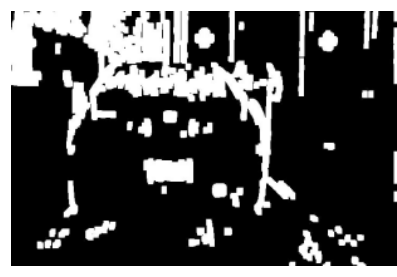

(f) Extracted number plate

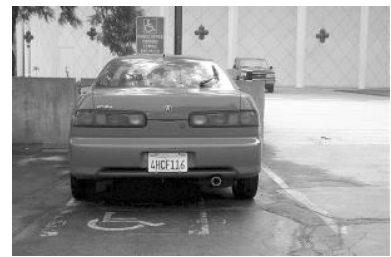

(b) Gray Image

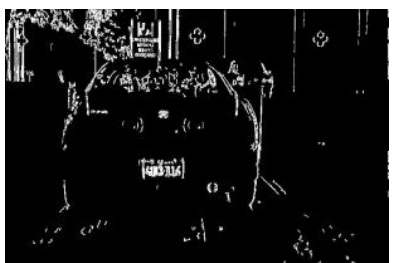

(d) Binary edge

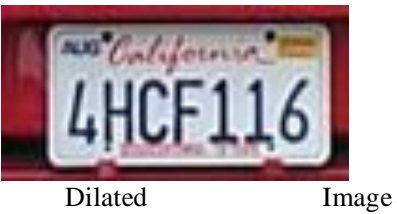

(e)

Fig. 7: (a) (b) (c) (d) (e) (f) Example of processed image.

Türky1lmaz, İ. and Kaçan, $\mathrm{K}$ in [11] their research work image processing algorithms are used to enhance image by improving system performance. The license plate location (rectangular shape) is fetched by utilizing edge based image processing approaches on the binarized image. The region of plate is prepared for the character segmentation with the help of skew correction phase. Vertical projection is used for characters separation. Character recognition is achieved by segmented characters with the help of thinning process. A three layer feedforwarded artificial Neural network utilizing back propagation learning algorithm is designed with the help of character recognition stage for 357 test images in data set taken under specified conditions. Results show a success rate of $97 \%$ with system processing time duration is $60 \mathrm{~ms}$ in all images.

Table 1: Performance of the LPRS

\begin{tabular}{|l|l|l|}
\hline LPRS stage & $\begin{array}{l}\text { Number of im- } \\
\text { ages/ success }\end{array}$ & Performance (\%) \\
\hline LP region determination & $357 / 357$ & 100 \\
\hline Character segmentation & $357 / 357$ & 100 \\
\hline Character recognition & $357 / 346$ & 96.92 \\
\hline
\end{tabular}

B. V. Kakani, D. Gandhi and S. Jani, [12] used a blend of algorithm for Localization \& computation of different OCR algorithm for recognition of license plate. After processing 300 images of different characters for training of neural network with input comprises of 30 neuron and output 36 neuron.

Table 2: Comparative analysis of different method for localization Algorithm.

\begin{tabular}{|c|c|c|c|}
\hline Algorithm & Accuracy & $\begin{array}{c}\text { Computation } \\
\text { Period }\end{array}$ & Comments \\
\hline $\begin{array}{c}\text { Projection } \\
\text { Technique }\end{array}$ & $89 \%$ & $0.91 \mathrm{~s}$ & $\begin{array}{c}\text { Effective for } \\
\text { different styles } \\
\text { of NP }\end{array}$ \\
\hline $\begin{array}{c}\text { Morphological } \\
\text { Operation }\end{array}$ & $96.7 \%$ & $0.54 \mathrm{~s}$ & $\begin{array}{c}\text { Effective on } \\
\text { noisy images }\end{array}$ \\
\hline $\begin{array}{c}\text { Feedback } \\
\text { selflearning } \\
\text { and } \\
\text { Hybrid } \\
\text { binarization }\end{array}$ & 97.1 & $0.51 \mathrm{~s}$ & $\begin{array}{c}\text { High } \\
\text { complexity, low } \\
\text { resolution } \\
\text { images required }\end{array}$ \\
\hline $\begin{array}{c}\text { Feature salience } \\
\text { Fearion }\end{array}$ & $97.3 \%$ & $0.23 \mathrm{~s}$ & $\begin{array}{c}\text { High resolution, } \\
\text { low noise } \\
\text { images required. }\end{array}$ \\
\hline
\end{tabular}

They have trained ANN for 300 iteration which took around 15 seconds to complete by using FFBPNN for learning and logsigmoid function at the hidden layer. The proposed algorithm designed after all the work gave an accuracy of $94.45 \%$ in less than 1 second is claimed.

Table 3: Different Calculations of OCR algorithms.

\begin{tabular}{|c|c|c|}
\hline Algorithm & $\begin{array}{c}\text { Computational time } \\
(\mathbf{m s )}\end{array}$ & $\begin{array}{c}\text { Detection rate } \\
(\%)\end{array}$ \\
\hline i-novel & 470 & $73 \%$ \\
\hline $\begin{array}{c}\text { Template } \\
\text { matching }\end{array}$ & 1000 & $94 \%$ \\
\hline Width analysis & 430 & $86 \%$ \\
\hline $\begin{array}{c}\text { ANN using } \\
\text { template } \\
\text { Matching }\end{array}$ & 176 (post training) & $89.4 \%$ \\
\hline $\begin{array}{c}\text { ANN using } \\
\text { feature } \\
\text { Extraction }\end{array}$ & $\mathbf{7 5}$ (post training) & $\mathbf{9 2 . 2 \%}$ \\
\hline
\end{tabular}

JosephTarigan Nadia Ryanda Diedan and YayaSuryana [17] they have used top-hat transformation and otsu threshold with Genetic Algorithm in optimising the number of hidden neuron, learning rate and momentum rate on BPNN that is applied on NPR. They analysed 220 photos with various lightening condition and plate condition as the sample for recognition. They found $85.97 \%$ success rate in whole characters recognition and $97.18 \%$ in single character recognition accuracy by using GA in BPNN.

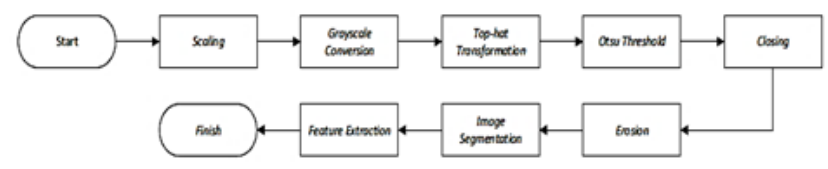

Fig 8: Pre-processing flow

N. Wang, X. Zhu and J. Zhang [18] have developed Set of Algorithms for Chinese Number plate's segmentation and recognition. Four algorithms of image pre-processing, number plate location, number plate segmentation and character recognition are developed. Specifically they have developed a formula for number plate 
location as per Chinese plate standard and use radon transform to correct license plate image. In this training model is generated using the character segmentation image by BPNN. Finally 11 Number plates have been correctly verified out of 13 which give $84.62 \%$ number plate recognition rate. Out of 91 characters 89 have been recognized correctly with gives $97.80 \%$ character recognition rate.

$$
\left[\begin{array}{l}
x^{\prime} \\
y^{\prime}
\end{array}\right]=\left[\begin{array}{rr}
\cos \theta & \sin \theta \\
-\sin \theta & \cos \theta
\end{array}\right]\left[\begin{array}{l}
x \\
y
\end{array}\right]
$$

(a)

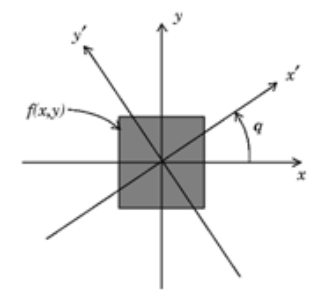

(b)
Fig 9: (a) (b) Radon Transform for number plate location

R. Shandilya and R. K. Sharma, [13] in used a simple and effective Image enhancement based on FPGA for Automatic Number Plate of vehicle detection. In this the most challenging problem for Number Plate identification is their image quality for all those images which are captured in low light during night, due to low visibility quality of all image got degraded and will be out of focus due to vehicle movement is considered as well. In this work techniques used for image enhancement such as contrast, brightness control and sharpness which are vital for noisy and blur image. The algorithm discussed has been implemented on Xilinx VirtexV FPGA device. For proposed implementation evaluated average process delay is around $4 \mathrm{~ns}$ with power $102 \mathrm{~mW}$.

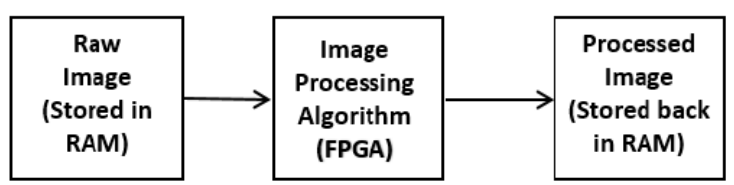

Fig10: Block diagram of Image Enhancement System

Table 4: Comparison of implemented architecture design with existing design

\begin{tabular}{|c|c|c|c|c|}
\hline Architecture & $\begin{array}{l}\text { M.Chandrashekar et. Al } \\
{[8]}\end{array}$ & Wan Bing-jian et. Al [11] & $\begin{array}{l}\text { Abduallah M. A. el. Al } \\
\text { [6] }\end{array}$ & Proposed \\
\hline Implementation & $\begin{array}{c}\text { Infrared Image } \\
\text { Enhancement using } \\
\text { Successive Mean } \\
\text { Quantization Transform }\end{array}$ & $\begin{array}{l}\text { Plateau Histogram } \\
\text { Equalization }\end{array}$ & Histogram Equalization & $\begin{array}{c}\text { Sharpness Control \& } \\
\text { Histogram Equalizatio }\end{array}$ \\
\hline Device & $\begin{array}{l}\text { Xilinx Virtex E } 2000 \\
\text { FPGA }\end{array}$ & $\begin{array}{l}\text { EP1K1100QC208 of } \\
\text { Altera Acexlk }\end{array}$ & $\begin{array}{l}\text { Stratix If family chip } \\
\text { type EP2S15F484C3 }\end{array}$ & $\begin{array}{c}\text { xc5vlx330-2ffl } 760 \\
\text { FPGA }\end{array}$ \\
\hline Area & $\begin{array}{l}\text { I/O cells } 34 \text { of } 454 \\
(7 \%) \text {, Block RAMs } 48 \text { of } \\
56(86 \%)\end{array}$ & $\begin{array}{c}\text { I/O cells } 25 \text { of } 160 \\
(40 \%), 32 \times 1 \text { RAMs } 256\end{array}$ & * & $\begin{array}{l}\text { I/O cells } 32 \text { of } 1200 \text { th } \\
\text { (2.6\%), Block RAMs } 800 \\
\text { of } 10,368(7.7 \%) \text { b }\end{array}$ \\
\hline Time Period & $*$ & $108.807 \mathrm{~ns}$ & $0.262 \mathrm{~ms}$ & $4 \mathrm{~ns}$ \\
\hline Power & * & & $*$ & $102 \mathrm{~mW}$ \\
\hline
\end{tabular}

S. S. Omran and J. A. Jarallah in [14] reported a system of automatic license plates identification among three different style Iraqi car license plate. Three styles of license plate were distinguished by their plate size. The templates matching and correlation approach was used with optical character recognition OCR for plate recognition by segmenting each character, number and word into sub images. For thresholding they have used Otsus mehod and morphological operations to enhance the image. The system is analyzed over 40 images and finally it gives $87.5 \%$ for extraction of plate region and approx $85.7 \%$ for the recognition unit accurate, giving the overall system performance of $86.6 \%$ recognition rate.

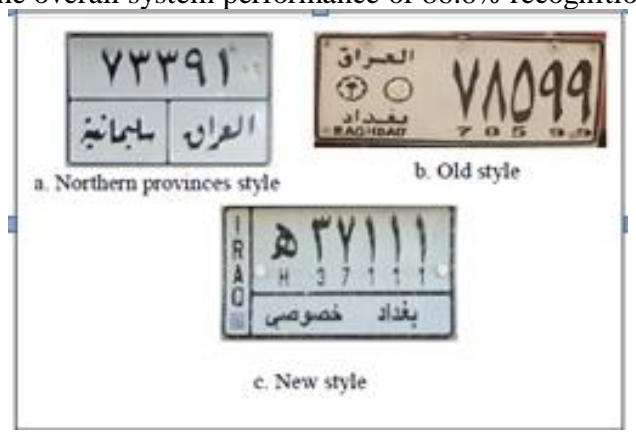

Fig.11 (a) Iraqi car license plate types

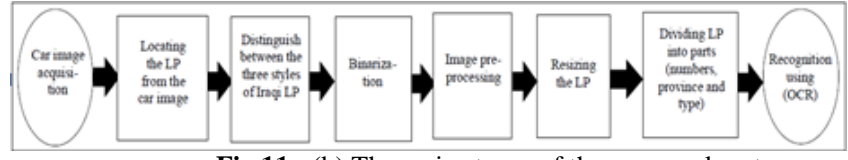

Fig.11: (b) The main stages of the proposed systemare

A. Y. Felix, A. Jesudoss and J. A. Mayan, in [15] used Adaptive Histogram Equalization, active contour method for region separation Optical Character Recognition to recognize the characters and Neural Network for Monitoring entry and exit using license plate recognition. They have used Deep Neural Network approach for classification and extracting the text as an alphanumeric character and comparing the text with the predefined table created in MYSQL server. In the proposed system they applied extraction of characters from the license plate and changing the status accordingly with comparing to the table works with an $100 \%$ accuracy level.

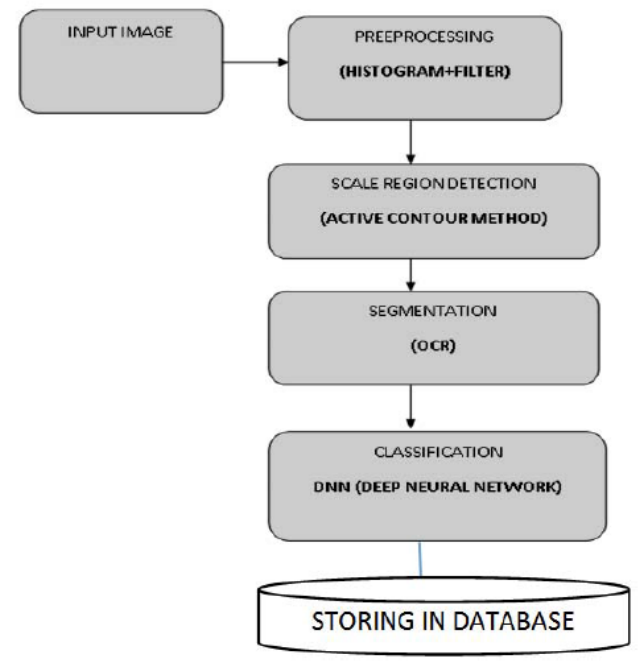

Fig 12: Block diagram of license plate recognition

A. Farhat et al., [16] have applied four algorithms for comparison of Qatari number plate's recognition system. Template matching and feature extraction such as (zoning, combined zoning and vector crossing) algorithms are used in this approach. A data set of 2790 segmented characters is used for all proposed algorithm ${ }^{60}$ hich are verified and tested on MATLAB. Outcome have shown the higest recognition rate of $99.5 \%$ for the algorithm which are based on template matching and utilizes average processing time of $1.95 \mathrm{~ms}$ for one image. It is observed that it is the most nose tolerant method and is the able to detect rotated characters. Which optimize the system complexity of the ANPR system as it might avoid the need for an extra pre-processing stage to adjust rotated Number Plates.

Table 5: Performance of Different OCR Algorithms

\begin{tabular}{|c|c|c|c|c|c|}
\hline \multicolumn{2}{|c|}{ OCR Technique } & \multicolumn{2}{|l|}{ Platform } & $\begin{array}{c}\text { Recognition } \\
\text { Rate [\%] }\end{array}$ & $\begin{array}{c}\text { Processing } \\
\text { Time per } \\
\text { character } \\
\text { [ms] }\end{array}$ \\
\hline \multirow{4}{*}{ 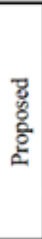 } & $\begin{array}{l}\text { Vector } \\
\text { crossing }\end{array}$ & \multirow{4}{*}{$\begin{array}{c}\text { Intel Core i7 } \\
-4770 \mathrm{~s}-3.1 \\
\mathrm{GHz} \\
8 \mathrm{~GB} \text { of } \\
\text { RAM }\end{array}$} & \multirow{7}{*}{$\sum_{j}^{\stackrel{m}{\xi}}$} & 90.43 & 1.33 \\
\hline & Zoning & & & 85.27 & 1.33 \\
\hline & $\begin{array}{l}\text { Zoning and } \\
\text { vector } \\
\text { crossing }\end{array}$ & & & 90.43 & 1.46 \\
\hline & Correlation & & & 99.50 & 1.95 \\
\hline \multirow{3}{*}{$\frac{\text { 总 }}{\overline{0}}$} & [1] & $\begin{array}{l}\text { Dual Core - } \\
2.4 \mathrm{GHz}-3 \\
\text { GB of RAM }\end{array}$ & & 97.3 & 8.4 \\
\hline & [6] & \multirow{2}{*}{$\begin{array}{c}\text { Intel Core i3 } \\
-2330 \mathrm{M}- \\
2.2 \mathrm{GHz}-2 \\
\text { GB of RAM }\end{array}$} & & 97 & $\begin{array}{c}\text { Not } \\
\text { available }\end{array}$ \\
\hline & [7] & & & 99 & $\begin{array}{c}\text { Not } \\
\text { available }\end{array}$ \\
\hline
\end{tabular}

Mahamad A.K in [10] proposed an automatic inspection system to recognise Malaysian vehicle plate number using Lab- 
VIEW software. In this paper they have used to approaches which contains two major modules. Firstly, to locate region of Interest (ROI) in captured image of license plate. Secondly, they have used concept of Optical Character Recognition (OCR) for Vehicle plate number recognition module to identify all individual character for each number plate which contains different alphanumerics. This system is tested for various implementations to ensure that the proposed method can be applied for real implementation. An intelligent OCR training interface has been used as library. The proposed system shows good performance for inspection and can recognise an alphabets and number of vehicle number plate.

Yo-Ping Huang, Shi-Yong Lai and Wei-Po Chuang [19] have designed a system to achieve automatic recognition of number plate which is intend to have high accuracy and reliability of number plate recognition. They reported a framework to identify the system of license number plate recognition in the captured image from a video. There are four major stages for recognition process. First, they have utilized gradient analysis and image processing approach which attempt to identify the most probable location of the license plate. Second, they have used cross-correlation to identify the tilt through skew correction of the license plate and it compute the vital image parameters which is required to normalize the position of license plate. Finally, for characters recognition they have utilized a template technique. Collectively all the previous steps are utilized to identify the probable license number plate. Hence the proposed system is successfully able to recognize the license number plate in only 1.5 seconds.

\section{Conclusion}

The main objective to discuss multiple techniques in this paper was to identify developments in Number plate recognition (NPR) system that involve fast processing, less computing power and which technique has best recognition rate with few restriction. Researchers have used Template matching, SVM, Canny edge detection, Neural Network, BPNN, Genetic Algorithm Feed forwarded Neural Network, fuzzy logic, FPGA to search for robust technique to recognize each character in number plate accurately. Undoubtedly in future Neural Network can bring high correctness rate with cent percent recognition accuracy. It ought to be observed that there are numerous commercial NPR systems whose analysis is beyond the scope in this research. That is only because of their commercial technology which is confidential and often results are overestimated for showoff purpose only. All the work which is discussed in this paper will surely support the practical performance evaluation to research forum globally and enables researchers to select which methods are best suited for which application in the field of number plate recognition.

\section{References}

[1] Bouchet A, Pastore J and Ballariu V, "Segmeutatiou of Medical images usiug Fuzzy Mathematical Morphology", JCS aud T, Vol7, No.3, Octorber 2007, pp. $256-262$.

[2] P.Cheu, G.Waug, Y.Yang and lZhou, "Facial expressiou Recoguitiou based ou Rough Set Theory and SVM", Lecture Notes iu

[3] Computer Science, Springer Berlin/Heidelberg, Rough Sets and Knowledge Technology, Vol. 4062, 2006 pp 772 -777.

[4] N. Otsu, "A threshold selection method from gray-level histograms," IEEE Trans. Syst., Man, Cybern., vol. SMC-9, no. 1, pp. 62-66, Jan. 1979.

[5] P. Kakkar and U. Dutta, "A novel Approach to Recognition of English Characters Using Artificial Neural Networks", International Journal of Advanced Research in Electrical, Electronics and Instrumentation Engineering, Vol 3, Issue 6, June 2014.

[6] F. Martin, M. Garcia, and J. L. Alba, "New methods for automatic reading of VLP's (Vehicle License Plates)," in Proc. IASTED Int. Conf. SPPRA, 2002

[7] X. Shi, W. Zhao, and Y. Shen, "Automatic License Plate Recognition System Based on Color Image Processing," Comput. Sci. Its Applicat., Singapore, May 9-12, 2005, pp. 1159-1168.
[8] R. Zunino and S. Rovetta, "Vector Quantization for License-Plate Location and Image Coding," IEEE Trans. Ind. Electron., vol. 47, no. 1, Feb. 2000, pp. 159-167.

[9] T.D. Duan et al., "Building an Automatic Vehicle License-Plate Recognition System," Proc. Int. Conf. Comput. Sci., Cantho, Vietnam, Feb. 2005, pp. 59-63.

[10] Y. Shima, "Extraction of number plate images based on image category classification using deep learning," 2016 IEEE International Symposium on Robotics and Intelligent Sensors (IRIS), Tokyo, 2016, pp. 19-26.

[11] Mahamad A.K., Saon S., Aziz S.N.O.A. (2014) A Simplified Malaysian Vehicle Plate Number Recognition. In: Herawan T., Ghazali R., Deris M. (eds) Recent Advances on Soft Computing and Data Mining. Advances in Intelligent Systems and Computing, vol 287. Springer, Cham

[12] Türkyılmaz, İ. and Kaçan, K. (2017), License Plate Recognition System Using Artificial Neural Networks. ETRI Journal, 39: 163 172.

[13] B. V. Kakani, D. Gandhi and S. Jani, "Improved OCR based automatic vehicle number plate recognition using features trained neura network," IEEE 2017 8th International Conference on Computing, Communication and Networking Technologies (ICCCNT), Delhi, 2017, pp. 1-6.

[14] R. Shandilya and R. K. Sharma, "FPGA implementation of image enhancement technique for Automatic Vehicles Number Plate detection," IEEE 2017 International Conference on Trends in Electronics and Informatics (ICEI), Tirunelveli, 2017, pp. 1010-1017 S. S. Omran and J. A. Jarallah, "Iraqi car license plate recognition using OCR," IEEE 2017 Annual Conference on New Trends in Information \& Communications Technology Applications (NTICT), Baghdad, 2017, pp. 298-303.

A. Y. Felix, A. Jesudoss and J. A. Mayan, "Entry and exit monitoring using license plate recognition," 2017 IEEE International Conference on Smart Technologies and Management for Computing, Communication, Controls, Energy and Materials (ICSTM), Chennai, 2017, pp. 227-231.

[16] Farhat et al., "OCR based feature extraction and template matching algorithms for Qatari number plate," IEEE 2016 International Conference on Industrial Informatics and Computer Systems (CIICS), Sharjah, 2016, pp. 1-5.

[17] JosephTarigan Nadia Ryanda Diedan and YayaSuryana " Plate Recognition Using Backpropagation Neural Network and Genetic Algorithm," Science Direct Procedia Computer Science Volume 116, 2017, Pages 365-372

[18] N. Wang, X. Zhu and J. Zhang, "License Plate Segmentation and Recognition of Chinese Vehicle Based on BPNN," 2016 12th International Conference on Computational Intelligence and Security (CIS), Wuxi, 2016, pp. 403-406

[19] Yo-Ping Huang, Shi-Yong Lai and Wei-Po Chuang, "A templatebased model for license plate recognition," IEEE International Conference on Networking, Sensing and Control, 2004, 2004, pp. 737$742 \mathrm{Vol} .2$ doi: 10.1109/ICNSC.2004.1297038

[20] D. Hongyao and S. Xiuli, "License Plate Characters Segmentation Using Projection and Template Matching," 2009 International Conference on Information Technology and Computer Science, Kiev, 2009, pp. 534-537 